
Not for reproduction, distribution or commercial use.

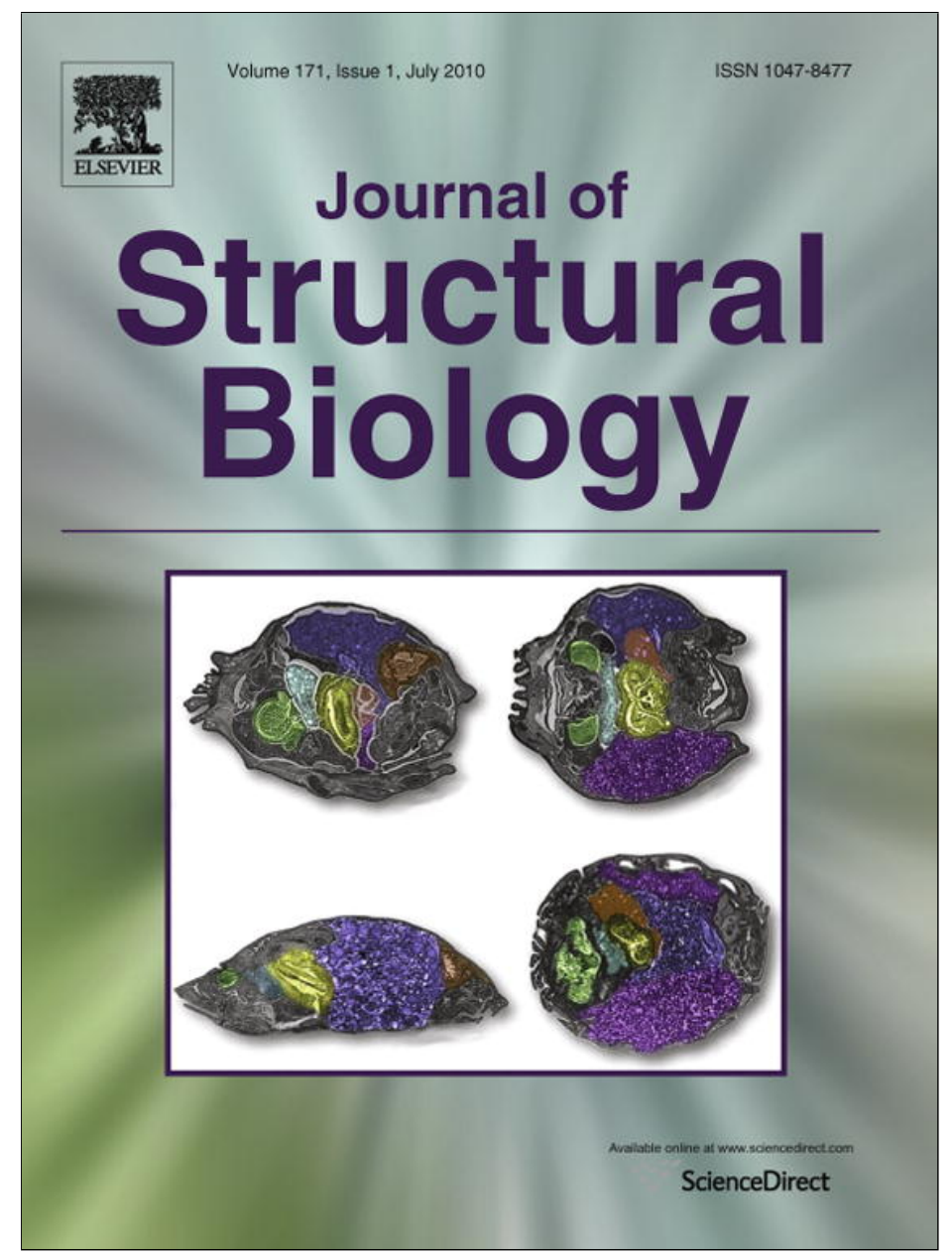

This article appeared in a journal published by Elsevier. The attached copy is furnished to the author for internal non-commercial research and education use, including for instruction at the authors institution and sharing with colleagues.

Other uses, including reproduction and distribution, or selling or licensing copies, or posting to personal, institutional or third party websites are prohibited.

In most cases authors are permitted to post their version of the article (e.g. in Word or Tex form) to their personal website or institutional repository. Authors requiring further information regarding Elsevier's archiving and manuscript policies are encouraged to visit:

http://www.elsevier.com/copyright 


\title{
Subtle structural differences between human and mouse PAI-1 reveal the basis for biochemical differences
}

\author{
Maarten Dewilde ${ }^{\mathrm{a}}$, Britt Van De Craen ${ }^{\mathrm{a}}$, Griet Compernolle ${ }^{\mathrm{a}}$, Jeppe B. Madsen ${ }^{\mathrm{a}, 1}$, Sergei Strelkov ${ }^{\mathrm{b}}$, \\ Ann Gils ${ }^{a}$, Paul J. Declerck ${ }^{\mathrm{a}, *}$ \\ ${ }^{a}$ Laboratory for Pharmaceutical Biology, Faculty of Pharmaceutical Sciences, Katholieke Universiteit Leuven, Belgium \\ ${ }^{\mathrm{b}}$ Laboratory for Biocrystallography, Faculty of Pharmaceutical Sciences, Katholieke Universiteit Leuven, Belgium
}

\section{A R T I C L E I N F O}

\section{Article history:}

Received 6 January 2010

Received in revised form 10 March 2010

Accepted 10 March 2010

Available online 15 March 2010

\section{Keywords:}

Plasminogen activator inhibitor-1

PAI-1

Serpin

Crystal structure

Rational drug design

\begin{abstract}
A B S T R A C T
Plasminogen activator inhibitor-1 (PAI-1) is a serine protease inhibitor (serpin) that plays an important role in cardiovascular disorders and tumor development. The potential role of PAI-1 as a drug target has been evaluated in various animal models (e.g. mouse and rat). Sensitivity to PAI-1 inhibitory agents varied in different species. To date, absence of PAI-1 structures from species other than human hampers efforts to reveal the molecular basis for the observed species differences.

Here we describe the structure of latent mouse PAI-1. Comparison with available structures of human PAI-1 reveals (1) a differential positioning of $\alpha$-helix A; (2) differences in the gate region; and (3) differences in the reactive center loop position. We demonstrate that the optimal binding site of inhibitors may be dependent on the orthologs, and our results affect strategies in the rational design of a pharmacologically active PAI-1 inhibitor.
\end{abstract}

(c) 2010 Elsevier Inc. All rights reserved.

\section{Introduction}

Plasminogen activator inhibitor-1 (PAI-1) is the most important physiological inhibitor of tissue-type plasminogen activator (t-PA) and urokinase-type plasminogen activator (u-PA) (Kruithof et al., 1984). It plays an important role in the regulation of the fibrinolytic activity in human blood (van Meijer and Pannekoek, 1995). The relationship between increased PAI-1 levels and various vascular disorders such as venous thrombo-embolism, coronary artery disease, myocardial infarction and atherosclerosis has been demonstrated (Prins and Hirsh, 1991; Juhan-Vague et al., 1995; Wiman, 1999). In addition to its role in fibrinolytic processes, PAI-1 has been demonstrated to be involved in a variety of other pathological processes (Durand et al., 2004; Binder and Mihaly, 2008). Elevated PAI-1 levels were also detected during wound healing, in rheumatoid arthritis, chronic stress, glomerulo-nephritis and gram-negative sepsis (Lijnen, 2005). A variety of studies have suggested that PAI-1 may play a role in tumor growth and metastasis. This effect could be related either to its vitronectin-binding properties (Declerck et al., 1988) or to its antiprotease properties (Bajou

\footnotetext{
* Corresponding author. Address: Laboratory for Pharmaceutical Biology, Faculty of Pharmaceutical Sciences, Katholieke Universiteit Leuven, Campus Gasthuisberg, O\&N2, Herestraat 49, PB 824, B-3000 Leuven, Belgium. Fax: +32 16323460.

E-mail address: paul.declerck@pharm.kuleuven.be (P.J. Declerck).

1 Present address: Department of Molecular Biology, University of Aarhus, Denmark.
}

et al., 1998). Furthermore, the importance of PAI-1 in vivo has been demonstrated in studies using transgenic mice lacking (Carmeliet et al., 1993) or overexpressing (Erickson et al., 1990) PAI-1.

PAI-1 is synthesized in the active form and binds to vitronectin in plasma and in the extracellular matrix (Declerck et al., 1988). PAI-1 spontaneously converts to the latent form under physiological conditions with a half-life ranging between 90 and $120 \mathrm{~min}$ (Hekman and Loskutoff, 1985). PAI-1 is a member of the serine protease inhibitor (serpin) superfamily and is a single-chain, glycosylated protein of approximately $50 \mathrm{kDa}$ (379 amino acid residues). Up to now, three different conformations of PAI-1 have been described: an active, a latent and a substrate conformation (Hekman and Loskutoff, 1985; Declerck et al., 1992; Mottonen et al., 1992; Munch et al., 1993; Sharp et al., 1999; Nar et al., 2000). The tertiary structure of active PAI- 1 shares motifs common to most serpins, including the solvent-exposed reactive centre loop (RCL) of about 20 amino acids long (designated P16 through P8', including the bait peptide bond Arg346-Met347 (P1-P1')) (Mottonen et al., 1992). In its active conformation, this P1-P1' reactive centre bond is cleaved upon reaction with a protease, followed by an insertion of the amino terminal part of the RCL into $\beta$-sheet A, hereby relocating the protease to the opposite pole of PAI-1 (Shore and Day, 1995). This results in the formation of a $1: 1$ SDS-stable acyl-enzyme complex (Egelund et al., 1998). During this remarkable conformational change, the active site of the protease is distorted, and, as a consequence, the protease is inhibited (Huntington et al., 2000; Dementiev et al., 2006). Upon latency 
conversion, the N-terminal part of the RCL from P16 to P3 is inserted into $\beta$-sheet $\mathrm{A}$, forming the new $\beta$-strand 4A. Residues P2 to $\mathrm{P}^{\prime}$ of the RCL form an extended loop after latency transition. The environment of the reactive center loop changes from solvent-exposed to mostly buried, yielding the reactive center (P1P1') inaccessible for the target proteases (Mottonen et al., 1992).

Several human PAI-1 structures have been reported. The first PAI-1 conformation that was structurally characterized was latent PAI-1, elucidated in 1992 by Mottonen et al. (1992) (PDB entry 1C5G). Subsequently, two other groups elucidated a structure of human PAI-1 in the latent conformation (Stout et al., 2000 (PDB entry 1DVN); Stein and Baek, 2002 (PDB entry 1LJ5)). The overall pairwise rms deviation between all three structures is $<0.45 \AA$, showing the high structural homology across those structures. These three structures originate from crystals with the same space group, i.e. $\mathrm{C}$. Three structures of mutants of human PAI- 1 in the active conformation have been published (Sharp et al., 1999 (PDB entry 1B3K); Nar et al., 2000 (PDB entry 1DB2); Stout et al., 2000 (PDB entry 1DVM)). All three structures are highly similar (pairwise rms deviation between all structures is $<0.6 \AA$ ). Two structures originate form a crystal with a $P 1$ space group, and one from a crystal with a $P 3_{1} 21$ space group. Three structures of cleaved, human PAI-1 variants have been reported (Aertgeerts et al., 1995 (PDB entry 9PAI); Jensen and Gettins, 2008 (PDB entry 3CVM); Dewilde et al., 2009 (PDB entry 3EOX)). In addition, crystal structures of human PAI-1 variants were elucidated in which PAI-1 is complexed with an inhibiting pentapeptide (corresponding to P14-P10) (Xue et al., 1998) (PDB entry 1A7C) or with the somatomedin B domain of vitronectin (Zhou et al., 2003) (PDB entry 10C0).

All reported PAI-1 structures are derived from human PAI-1. However, since PAI-1 is considered as an important potential drug target in various diseases, its role has been evaluated in various animal models, including mouse, rat and rabbit. Rodents are commonly used as research models because they are readily available and relatively inexpensive. Disease pathogenesis in these animals generally tends to approximate disease progression in humans, although there is some divergence in cellular mechanism and pathogenesis (reviewed in Narayanaswamy et al. (2000) and Mestas and Hughes (2004)). In addition, most animal proteins are not identical to human proteins. Comparative evaluation of the putative therapeutic targets of different species is therefore of importance. The amino acid sequence identity between human PAI-1 (huPAI-1) and mouse PAI-1 (moPAI-1) is 78.9\% (Fig. 1). Although the stability and functional properties of PAI- 1 of both species are comparable (Xu et al., 2004), important species differences have been observed with respect to their sensitivity to PAI-1 inhibitory agents (Cale et al., 2010). The lack of structures of PAI- 1 from other species has hampered efforts to unravel the molecular basis for some of the observed species differences.

In this study, we describe the elucidation of the structure of mouse PAI-1 (moPAI-1). A detailed comparison of this moPAI-1 structure with available structures of human PAI-1 (huPAI-1) reveals three regions that are different between PAI-1 from these two species. Some of these structural differences provide a molecular explanation for previously described biochemical differences.

\section{Materials and methods}

\subsection{Production of moPAI-1 and antibodies}

Recombinant non-glycosylated moPAI-1 was expressed and purified in a similar way as huPAI-1-wt described in Gils et al. (1996). After purification, moPAI-1 was dialyzed against $50 \mathrm{mM}$

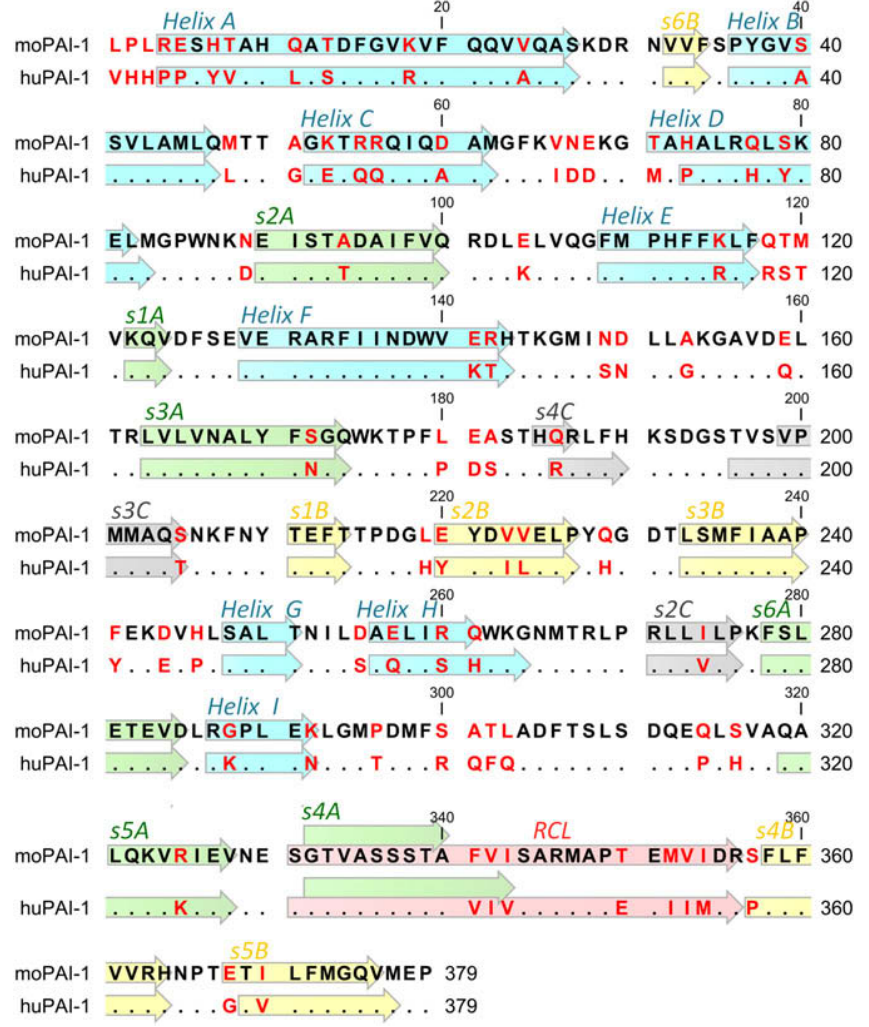

Fig. 1. Amino acid sequence alignment of mouse and human PAI-1. Different amino acids are indicated in red; secondary structure elements are indicated by the respective arrows; the $\mathrm{RCL}$ is indicated by the pink arrow. (For interpretation of the references to colour in this figure legend, the reader is referred to the web version of this article.)

HEPES, pH 7.5, and $0.4 \mathrm{M} \mathrm{NaCl}$. Mouse monoclonal antibody MA159M12 was produced as described elsewhere (Ngo et al., 2002).

\subsection{Crystallization}

Mouse PAI-1 samples were concentrated prior to crystallization using a Vivaspin 15R (cutoff $10 \mathrm{kDa}$ ) concentrator. To determine initial crystallization conditions, a selection of commercially available screens was applied (Hampton Research Crystal Screen 1 \& 2, Hampton Research Index Screen 1 \& 2, Emerald Biosystems Wizard Screen $1 \& 2$ ). The experimental setup was the hanging-drop vapor-diffusion geometry using Linbro multi-well tissue plates, stored at $277 \mathrm{~K}$ and/or $293 \mathrm{~K}$. Each well was filled with $700 \mu \mathrm{l}$ of reservoir solution and each hanging drop consisted of $1.5 \mu \mathrm{l}$ protein solution and $1.5 \mu \mathrm{l}$ of reservoir solution, placed on coverslips and set to equilibrate against these reservoir solutions. After 4 months, one crystal $(400 \times 200 \times 50 \mu \mathrm{m})$ appeared in condition 81 of Hampton Research Index Screen $(0.2 \mathrm{M}$ Ammonium acetate, 0.1 M Tris, pH 8.5, 25\% w/v PEG 3350, 277 K).

\subsection{Data collection and data processing}

Prior to flash-cooling in liquid nitrogen, the crystal was soaked for $30 \mathrm{~s}$ in a drop containing the crystallization solution to which $20 \%$ glycerol had been added. Diffraction data collection was carried out at the SLS synchrotron (Villigen, Switzerland) at $100 \mathrm{~K}$ using a liquid-nitrogen stream. A complete diffraction dataset could be collected to a resolution limit of $1.93 \AA$ (Table 1). Data were processed using XDS and XSCALE (Kabsch, 1993). The asymmetric unit contains one monomer, with a Matthews coefficient of 
Table 1

Data collection and refinement statistics for latent moPAI-1.

\begin{tabular}{|c|c|}
\hline \multicolumn{2}{|l|}{ Data collection } \\
\hline Wavelength $(\AA)$ & 0.9787 \\
\hline Resolution range $(\AA)$ & $40-1.93(1.98-1.93)$ \\
\hline \multicolumn{2}{|l|}{ Reflections } \\
\hline - Observed & 225,021 \\
\hline • Unique & 34,894 \\
\hline Completeness (\%) & $97.0(95.5)$ \\
\hline Average $I / \sigma$ & $20.2(4.3)$ \\
\hline$R_{\mathrm{sym}}(\%)$ & $6.8(47.4)$ \\
\hline \multicolumn{2}{|l|}{ Crystal } \\
\hline Space group & $\mathrm{C} 2$ \\
\hline Cell dimensions $\left(\AA,^{\circ}\right)$ & $\begin{array}{l}a=150.9, b=62.3, c=52.2, \\
\alpha=90.0, \beta=105.5, \gamma=90.0\end{array}$ \\
\hline \multicolumn{2}{|l|}{ Refinement } \\
\hline \multicolumn{2}{|l|}{ Number or atoms } \\
\hline - Protein & 2926 \\
\hline - Solvent & 227 \\
\hline \multicolumn{2}{|l|}{ Average B-factor $\left(\AA^{2}\right)$} \\
\hline - Protein & 31.6 \\
\hline - Solvent & 41.4 \\
\hline$R_{\text {work }}(\%)$ & 18.3 \\
\hline$R_{\text {free }}(\%)$ & 21.8 \\
\hline Reflections in the free' set & 1704 \\
\hline \multicolumn{2}{|l|}{ Rms deviations } \\
\hline - Bond lengths $(\AA ̊)$ & 0.011 \\
\hline - Bond angles $\left({ }^{\circ}\right)$ & 1.361 \\
\hline $\begin{array}{l}\text { Ramachandran plot (\% residues in } \\
\text { favoured/disallowed regions) }\end{array}$ & $98.9 / 0$ \\
\hline Molprobity score & 1.9 \\
\hline
\end{tabular}

Values in parentheses are for the highest resolution shell.

$2.63 \AA^{3} \mathrm{Da}^{-1}$ and a solvent content of $53 \%$. Further statistics is summarized in Table 1 . The structure was phased by molecular replacement in program PHASER using latent huPAI-1 (PDB entry $1 \mathrm{DVN}$ ) as a search model (McCoy et al., 2007). Five percent of the reflections were chosen at random as the test set for cross-validation during refinement. The initial search model was rebuild automatically using ARP/wARP 7.0.1. Model rebuilding included the adaptation of the huPAI-1 sequence to the moPAI-1 sequence (Fig. 1). Further model optimization and restrained refinement were done with COOT and REFMAC5, respectively (Emsley and Cowtan, 2004; Murshudov et al., 1997). During refinement, translation, libration and screw-rotation (TLS) corrections were applied (one TLS group). For the final refined structure, $98.9 \%$ of all residues are located in the Ramachandran favored regions and all residues are in the allowed regions. The final refinement statistics is shown in Table 1. The final model was verified using Molprobity (Davis et al., 2007), yielding an excellent Molprobity score (Table 1 ).

The final atomic model and diffraction data have been submitted to the Protein Data Bank under the accession code 3LW2.

\subsection{Construction and expression of moPAI-1 mutants}

MoPAI-1-R4P, moPAI-1-D150H-K154T-Q319L and moPAI-1I91L were constructed using appropriate mutagenic primers. The expression plasmid pIGE2-moPAI-1-wild-type (moPAI-1-wt) was used as template. Site-directed mutagenesis was performed using the QuikChange ${ }^{\mathrm{TM}}$ protocol (Stratagene, La Jolla, USA). Thermocycling was conducted in an Eppendorf Mastercycler ${ }^{\mathrm{TM}}$ gradient thermal cycler, using $50 \mathrm{ng}$ of template and $20 \mu \mathrm{M}$ of each mutant primer. Denaturation, annealing and elongation were carried out at $94{ }^{\circ} \mathrm{C}, 55.6{ }^{\circ} \mathrm{C}$ and $72{ }^{\circ} \mathrm{C}$, respectively. Subsequently, parental template DNA was removed by DpnI and the mutated DNA was transformed into DH1 $1 \lambda$ Escherichia coli cells, followed by overnight selection on agar plates containing tetracycline $(10 \mu \mathrm{g} / \mathrm{ml})$. For large-scale DNA purification, a single colony was selected and grown overnight in LB containing tetracycline $(10 \mu \mathrm{g} / \mathrm{ml})$ and the
DNA isolated using the Nucleobond ${ }^{\mathrm{TM}}$ AX 500 purification kit. The presence of the introduced mutation as well as the absence of any unwanted mutations was verified by nucleotide sequencing. The expression and purification was similar as for moPAI-1-wt.

\subsection{Characterization of moPAI-1 mutants}

\subsubsection{Affinity measurements}

Binding measurements were performed by surface plasmon resonance (SPR) analysis using BIAcore ${ }^{\mathrm{TM}} 3000$ (Biacore AB, Uppsala, Sweden). The carboxymethylated dextran matrix of the CM5 sensor chip (CM5) was preactivated using $N$-hydroxysuccinimide (NHS)/1-ethyl-3-[3-(dimethylaminopropyl) carbodiimide hydrochloride (EDC). Then, random amine coupling of the monoclonal antibody to the chip was carried out by injecting the antibody at a concentration of $5 \mu \mathrm{g} / \mathrm{ml}(10 \mathrm{mM}$ sodium acetate, $\mathrm{pH} 4.5)$ with a flow rate of $5 \mu \mathrm{l} / \mathrm{min}$ at $25^{\circ} \mathrm{C}$, Subsequently, residual NHS esters were deactivated by ethanolamine $(1 \mathrm{M}, \mathrm{pH} 8.5)$. The antibodies were covalently coupled to approximately 1200 resonance units. One RU corresponds to $1 \mathrm{pg}$ of bound protein $/ \mathrm{mm}^{2}$. PAI- 1 was diluted in HBS-EP buffer (Biacore) to concentrations between 5 and $200 \mathrm{nM}$ and injected at a flow rate of $30 \mu \mathrm{l} / \mathrm{min}$ at $25^{\circ} \mathrm{C}$. Association and dissociation data were both collected for $6 \mathrm{~min}$. After each cycle, the chip was regenerated with $10 \mu$ of $10 \mathrm{mM}$ glycine, $\mathrm{pH}$ 1.5. Analysis of the association and dissociation rates was performed using BIAevaluation version 3.1 software (Langmuir binding model, local fit) (Biacore). Data obtained from parallel flow cells to which a monoclonal antibody against TAFI (MA-T1C10) was coupled, served as blank sensorgrams for substraction of changes in the bulk refractive index.

\subsubsection{Functional stability}

PAI- 1 variants were diluted to concentrations between 0.1 and $0.2 \mathrm{mg} / \mathrm{ml}$ in the appropriate dilution buffer (final concentration; $0.025 \mathrm{M} \mathrm{Na}_{2} \mathrm{HPO}_{4}$ and $0.01 \%$ Tween $80, \mathrm{pH} 7.2-7.4$ ). The sample was incubated at $37{ }^{\circ} \mathrm{C}$ and aliquots removed at various time points. Subsequently, aliquots were incubated $\left(30 \mathrm{~min}, 37^{\circ} \mathrm{C}\right)$ with a 2-fold molar excess of tPA. Samples were subjected to SDS-PAGE (10-15\% gels, non-reducing environment) and proteins were visualized by staining with Coomassie Brilliant Blue. The conformational distribution (i.e. active/latent/substrate forms of PAI-1) (Gils and Declerck, 1997) was determined by densitometric scanning using Labscan ${ }^{\mathrm{TM}}$ and Imagemaster ${ }^{\mathrm{TM}}$ (GE Healthcare, Milwaukee, USA). The amount of active PAI- 1 at various time points was expressed as a percentage of the initial amount of active PAI-1 and the decay was used for the calculation of the half-life. Subsequently, the half-lives for inactivation were calculated with the program Graph Pad Prism ${ }^{\mathrm{TM}}$ using "one phase exponential decay" according to the equation: $Y=\operatorname{span} \times e-K \times X+$ plateau. The half-life is then equal to $0.693 / \mathrm{K}$.

To study the effect of MA-159M12 on the half-life, moPAI-1 variants were incubated with either an equal volume of PBS or a 3-fold molar excess of MA-159M12 at $37{ }^{\circ} \mathrm{C}$.

\section{Results and discussion}

\subsection{Structural analysis}

Since the crystallization took four months, the crystallized moPAI-1 was, not surprisingly, in the latent conformation. The final electron density was of good quality throughout the model, except for residues 346-356 (part of the RCL). However, as there was sufficient experimental evidence about the conformation of these residues, they were included in the final model (Fig. 2). Also (Mottonen et al., 1992) observed higher B-factors for these residues in 
latent huPAI-1, indicating a less pronounced electron density. For residues 1-6 and 189-196, there was no observable electron density at all, and hence these residues were omitted from the final latent moPAI-1 structure. The rms deviation of $C_{\alpha}$ positions between the refined latent moPAI-1 structure and latent huPAI-1 (PDB entry $1 \mathrm{DVN}$ ) is $0.786 \AA$, indicating a high structural homology between the two structures. However, superimposition of these two structures shows some local differences (Fig. 3).

\section{2. $\alpha$-Helix A region}

The first region that differs substantially between latent human and mouse PAI- 1 is the $\alpha$-helix A (hA) (Fig. 3). In all latent huPAI-1 structures (PDB entries 1C5G, 1DVN and 1LJ5), hA is located in exactly the same position, which is clearly distinct from the position in latent moPAI-1. Strikingly, the position of hA of latent moPAI-1 corresponds to the position of the hAs of all crystallized active huPAI-1 monomers (PDB entries 1B3K, 1DB2 and 1DVM) (Fig. 4). The first six amino acids of moPAI-1, corresponding to the N-terminus of hA, could not be built in the final model due to poor electron density maps for this region. Also the N-termini in all active huPAI-1 crystal structures are not clearly defined. Although some of the six N-terminal residues were built in for 1DB2 and 1DVM, their B-factors (up to $100 \AA^{2}$ ) suggest that these residues were not built in unambiguously. In latent huPAI- 1 , the N-terminal end of hA is bound to $\alpha$-helix D (hD) by hydrophobic (P4-A74; P5-L78) and electrostatic interactions (H3-E81). These interactions are not possible in latent moPAI-1 where position three harbors a leucine, position four an arginine and position five a glutamic acid. Consequently, the lack of binding of hA to hD in moPAI-1 will render hA more flexible, resulting in a lack of well-defined electron density in the crystals. It remains unclear why hA of active huPAI-1 is not attached to hD, despite the full sequence and structural homology in this region for active and latent huPAI-1. Also the C-terminal part of hA has a different conformation in both structures. It should be noted that the crystal packing arrangement of mouse PAI- 1 is different from that of the latent human PAI-1 structure as exemplified by the PDB structure 1DVN and may explain in part the observed differences with regard to both the $\mathrm{N}$ - and C-termini of helix A in both structures.

Previously, the epitope of a non-inhibitory monoclonal antibody raised against huPAI- 1 was mapped to the $\mathrm{N}$-terminal region of $\mathrm{hA}$ of huPAI-1 (MA-31C9; residues H3, S6, Y7 and H10) (Gils et al., 2009).

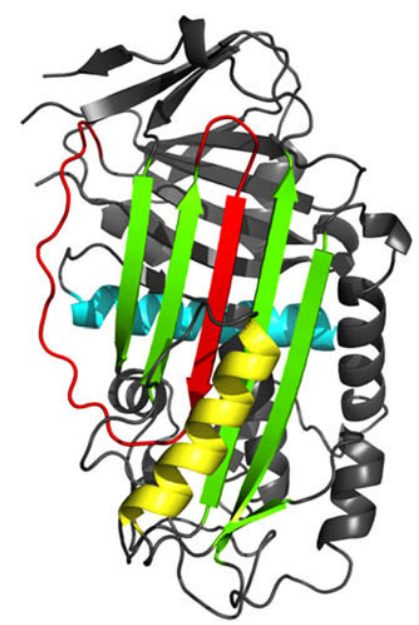

Fig. 2. Cartoon representation of moPAI-1. Beta sheet $A$ is indicated in green (s1As6A from right to left), $\alpha$-helix A in blue, $\alpha$-helix $F$ in yellow and the reactive centre loop is shown in red.
Surprisingly, this region overlaps with the epitope of another, but inhibitory, monoclonal antibody (i.e. MA-159M12) that was raised against rat PAI-1 $\left(K_{\mathrm{A}}=3.0 \pm 1.8 \times 10^{9} \mathrm{M}^{-1}\right)$ but reacts with a low affinity with moPAI-1 $\left(K_{\mathrm{A}}=0.07 \pm 0.055 \times 10^{9} \mathrm{M}^{-1}\right)$ (Ngo et al., 2002). MA-159M12 binds to P2, L3, P4 and E5, located in hA, and exerts a pronounced inhibitory effect by accelerating the active to latent conversion of rat PAI-1. Thus, while sharing an epitope region, antibodies MA-31C9 and MA-159M12 exert opposite functional effects (i.e. non-inhibitory vs. inhibitory). Rat and moPAI-1 share an amino acid sequence homology of $93.4 \%$ (sequence identity of $89.4 \%$ ). Based on this very high sequence homology, it is likely that both structures are highly similar, including hA. MoPAI- 1 harbors P2, L3 and E5 as in rat PAI-1. To study the effects of MA-159M12 on moPAI-1, a proline at position four was introduced (moPAI-1$\mathrm{R} 4 \mathrm{P}$ ) to have all four important residues contributing to the epitope of MA-159M12. As hypothesized moPAI-1-R4P exhibited an increased affinity towards MA-159M12 $\left(K_{\mathrm{A}}=0.52 \pm 0.14 \times 10^{9} \mathrm{M}^{-1}\right)$ and was susceptible to inhibition by MA-159M12 through a decrease of the half-life $(42 \pm 8.4 \mathrm{~min}$ in the presence of a 3 -fold molar excess of MA-159M12 vs. $107 \pm 3.5 \mathrm{~min}$ in the absence of MA-159M12). Previous attempts to introduce the epitope of MA-159M12 into hA of huPAI-1 did not succeed as this variant (huPAI-1-H2P-H3L-P5E) exhibited an affinity (i.e. $K_{\mathrm{A}}=3.4 \times 10^{6} \mathrm{M}^{-1}$ ) that is three orders of magnitude lower than that of rat PAI-1 (Gils et al., 2009). Taken together all available data show that the position of hA is similar in rat and mouse PAI- 1 but different compared to that of hA in huPAI-1, consequently resulting in different functional effects of monoclonal antibodies that bind to this region: i.e. inhibitory when binding to hA of mouse or rat PAI-1 vs. non-inhibitory when binding to hA of huPAI-1.

\subsection{Gate region}

The second region in moPAI- 1 with a different conformation compared to huPAI-1 is the gate region (the loop connecting $\beta$-strand $s 3 C$ to $\beta$-strand $s 4 C$ ) (Fig. 3). This region is quite well defined in all huPAI-1 structures whereas no electron density was observed for this region in moPAI-1. This is indicative for a much higher flexibility of the gate region in moPAI- 1 compared to huPAI-1. It is of interest to note that the gate region plays a role in the active to latent transition (Aertgeerts et al., 1995; Engh et al., 1993). The increased flexibility of the gate region in moPAI-1 may be related to the observation that mutations known to stabilize huPAI-1 do not have a similar effect on moPAI-1. To test this hypothesis, mutations that stabilize active human PAI-1 (i.e. N150H-K154T-Q319L-M354I) (Berkenpas et al., 1995) were introduced into mouse PAI-1 (resulting in moPAI-1-D150HK154T-Q319L, since moPAI-1 has already an Ile at position 354). HuPAI-1-N150H-K154T-Q319L-M354I has a 72-fold increased half-life $(145 \mathrm{~h})$, compared to huPAI-1-wt $(2 \mathrm{~h})$. However, introduction of the corresponding mutations in moPAI- 1 resulted only in a 2 -fold increased half-life (i.e. $1.9 \mathrm{~h}$ vs. $3.6 \mathrm{~h}$ for moPAI-1-wt and moPAI-1-D150H-K154T-Q319L, respectively) (Table 2). Alternatively a single mutation (Ile at position 91 to Leu) resulting in 9-fold increased half-life in huPAI-1 (huPAI-1-I91L, 18.4 h; (Berkenpas et al., 1995) did not affect the stability of mo-PAI-1 (i.e. half-life of moPAI-1-I91L, $2.1 \mathrm{~h}$ ). It should be noted that the structural environment of mutations in both positions 150 and 154 is virtually identical in mouse and human PAI- 1 . However, residue 319 adopts the same backbone position but its side chain is interacting with the RCL for which substantial structural differences are observed (see below). Finally, the environment of residue 354 is distinctly changed as this residue is located in the RCL. Taken together, the observed differential effects of these mutations are indicative for relatively small structural differences associated 


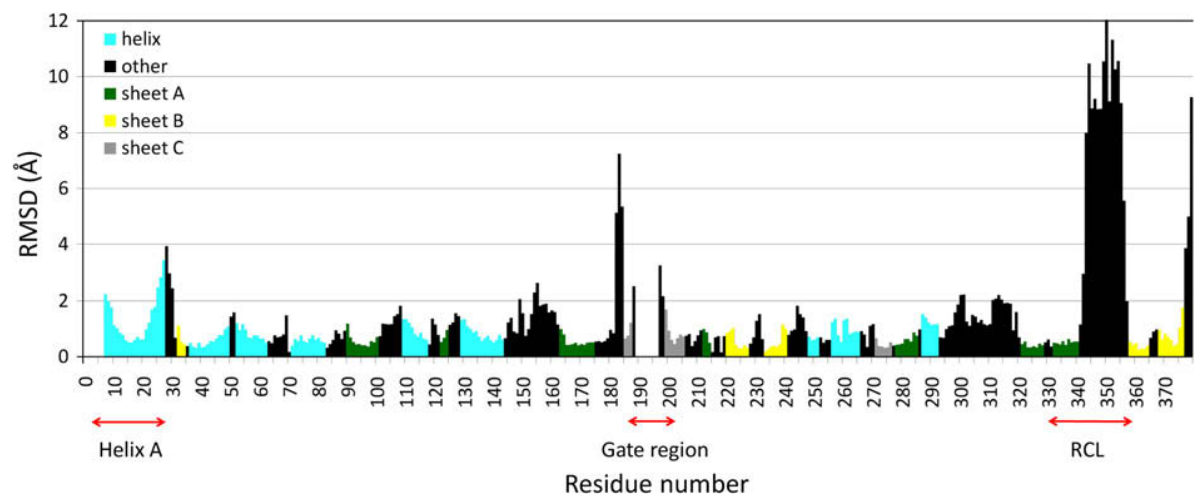

Fig. 3. Rms deviation between latent mouse PAI-1 and latent human PAI-1 vs. residue number. Ambiguous residues of the RCL were omitted.

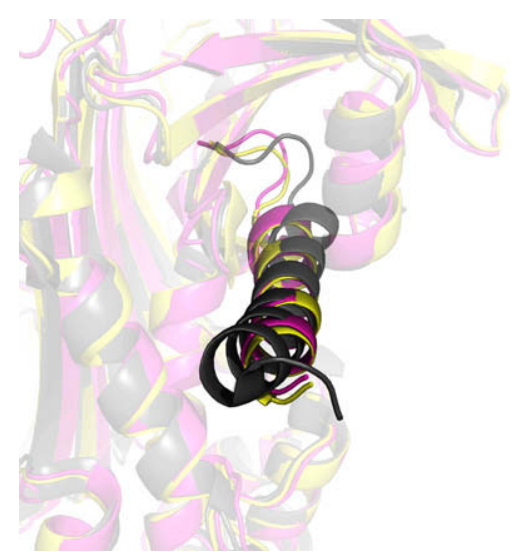

Fig. 4. Comparison of $\alpha$-helix A. Latent human PAI-1 (PDB entry 1DVN) is represented in gray, latent moPAI-1 in yellow, active human PAI-1 (PDB entry $1 \mathrm{BK} 3$, chain A) in pink.

Table 2

Half-lives (h) of mouse PAI-1 mutants vs. human PAI-1 mutants.

\begin{tabular}{llcc}
\hline & Wild-type & I91L & $150 / 154 / 319 /(354)^{\mathrm{a}}$ \\
\hline Mouse & $1.9 \pm 0.3$ & $2.1 \pm 0.2$ & $3.6 \pm 1.0$ \\
Human & 2.1 & $18.4 \pm 1.1^{\mathrm{b}}$ & $145.4 \pm 1.0^{\mathrm{b}}$ \\
\hline
\end{tabular}

a Mutation D150H-K154T-Q319L(-M354I) (see text).

b Data taken from Berkenpas et al. (1995).

with important differences in the properties of active moPAI-1 and active huPAI-1 (e.g. as observed for the gate region in this study).

\subsection{Reactive center loop}

The third region with structural differences comprises the RCL (Fig. 3). Although the central part of the RCL exhibits increased temperature factors compared with the rest of the structure, the $\mathrm{N}$ - and C-terminal portions of the RCL are well defined. Both portions differ substantially from the conformation observed in latent huPAI-1. N-terminally: the last residue of $s 4 \mathrm{~A}$ in latent moPAI-1 is F341 whereas in latent huPAI-1 s4A stretches till residue V343 (Fig. 5). C-terminally: the RCL is situated above of the C-terminus in latent moPAI-1, whereas this is the other way around in latent huPAI-1 (Fig. 6A). With the RCL being in this position in latent moPAI-1, it is impossible for the gate region in latent moPAI- 1 to adopt the conformation as observed in latent huPAI-1, as it would clash with the RCL (Fig. 6B). The different position of the RCL is also correlated to the different conformation of the C-terminal part of

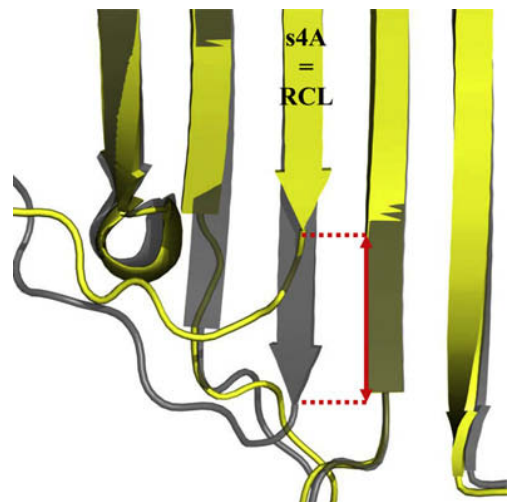

Fig. 5. Shorter $\beta$-strand $s 4 A$ in moPAI-1. Latent moPAI-1 is indicated in yellow, latent human PAI-1 in gray. In latent moPAI-1, a smaller portion of the RCL is inserted as $\mathrm{s} 4 \mathrm{~A}$, indicated in red.

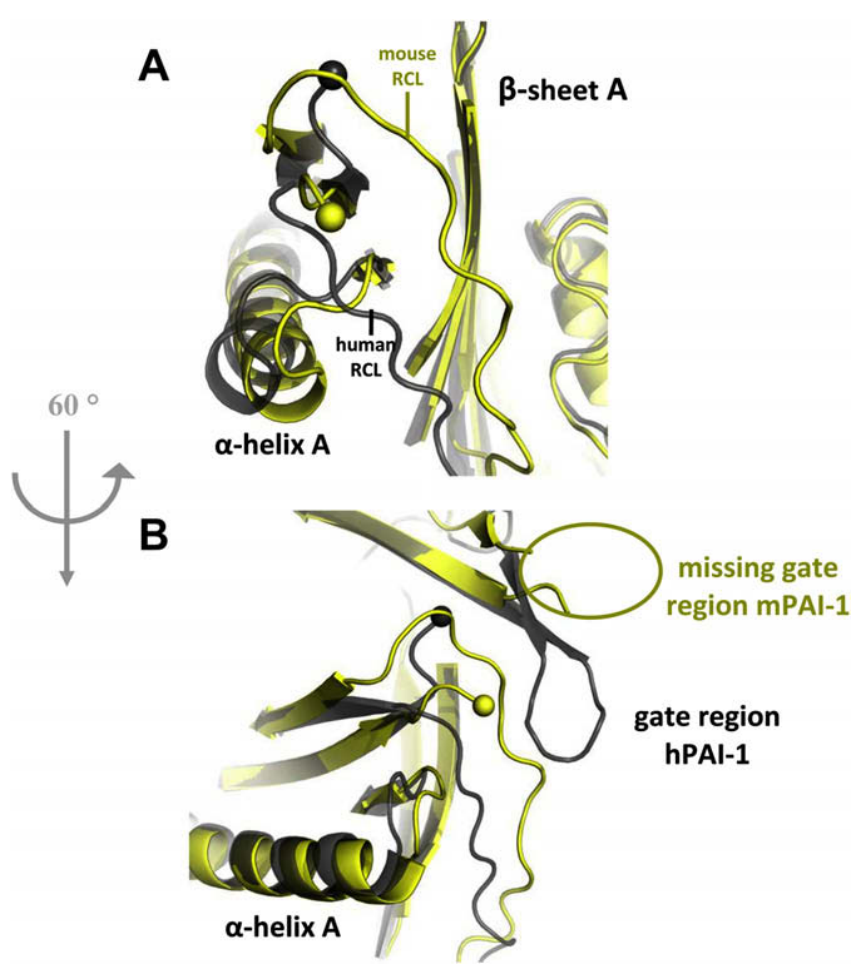

Fig. 6. C-terminal portion of the RCL. Two different views related by a $60^{\circ}$ rotation are shown. Latent moPAI-1 is indicated in yellow, latent human PAI-1 in gray. The C-termini of both PAI-1 structures are represented as spheres. For reasons of clarity the gate region is omitted in $(\mathrm{A})$. 
$\mathrm{hA}$ : in latent moPAI-1 hA is bent more to the core of the protein compared to hA in latent huPAI-1 (Fig. 6A).

It is worth mentioning that another study (Xu et al., 2004) reported a functional analysis of mutations in mouse PAI-1 demonstrating that a mutation in the vitronectin-binding domain (Q123K) or a mutation in the LRP-binding domain (R76E) of mouse PAI-1 abolished binding to vitronectin or LRP, respectively. Thus, these mutations yield identical effects in mouse as in human PAI-1. This is also in line with our current structural comparison, i.e. residues 73 and 123 are located in a region that is structurally not different between mouse and human PAI-1.

In conclusion, although similar to human PAI-1, the elucidated structure of moPAI-1 revealed some significant local differences. Importantly, the observed structural differences could be confirmed by biochemical observations. This may have important consequences in the rational design of LMW compounds for inhibition of PAI-1. Indeed the current study demonstrates that extrapolation of data for PAI-1 inhibition obtained in murine models should be done with caution, as the optimal binding site of a LMW inhibitor may be different for the different orthologs. In addition, this new PAI-1 structure contributes to the knowledge on the structurefunction relationship in PAI-1. Detailed structural insights into this therapeutic target are indispensable for the optimal design of a pharmacologically active PAI-1 inhibitor.

\section{Conflict of interest}

The authors state that they have no conflict of interest.

\section{Acknowledgments}

This study was supported in part by Grant G.0540.08 from the Fund for Scientific Research (FWO-Vlaanderen). M.D. and B.V.D.C. are Research Assistant of the Fund for Scientific Research (FWOVlaanderen).

\section{References}

Aertgeerts, K., De Bondt, H.L., De Ranter, C.J., Declerck, P.J., 1995. Mechanisms contributing to the conformational and functional flexibility of plasminogen activator inhibitor-1. Nat. Struct. Biol. 2, 891-897.

Bajou, K., Noel, A., Gerard, R.D., Masson, V., Brunner, N., Holst-Hansen, C., Skobe, M. Fusenig, N.E., Carmeliet, P., Collen, D., Foidart, J.M., 1998. Absence of hos plasminogen activator inhibitor 1 prevents cancer invasion and vascularization. Nat. Med. 4, 923-928.

Berkenpas, M.B., Lawrence, D.A., Ginsburg, D., 1995. Molecular evolution of plasminogen activator inhibitor-1 functional stability. EMBO J. 14, 2969-2977.

Binder, B.R., Mihaly, J., 2008. The plasminogen activator inhibitor "paradox" in cancer. Immunol. Lett. 118, 116-124.

Cale, J.M., Li, S.H., Warnock, M., Su, E.J., North, P.R., Sanders, K.L., Puscau, M.M., Emal, C.D., Lawrence, D.A., 2010. Characterization of a novel class of polyphenolic inhibitors of plasminogen activator inhibitor-1. J. Biol. Chem. 285, 7892-7902.

Carmeliet, P., Stassen, J.M., Schoonjans, L., Ream, B., van den Oord, J.J., De Mol, M., Mulligan, R.C., Collen, D., 1993. Plasminogen activator inhibitor-1 gene deficient mice. II. Effects on hemostasis, thrombosis and thrombolysis. J. Clin. Invest. 92, 2756-2760.

Davis, I.W., Leaver-Fay, A., Chen, V.B., Block, J.N., Kapral, G.J., Wang, X., Murray, L.W., Arendall III, W.B., Snoeyink, J., Richardson, J.S., Richardson, D.C., 2007. MolProbity: all-atom contacts and structure validation for proteins and nucleic acids. Nucleic Acids Res. 35, W375-W383.

Declerck, P.J., De Mol, M., Alessi, M.C., Baudner, S., Paques, E.P., Preissner, K.T., Muller Berghaus, G., Collen, D., 1988. Purification and characterization of a plasminogen activator inhibitor 1 binding protein from human plasma. Identification as a multimeric form of S protein (vitronectin). J. Biol. Chem. 263, 15454-15461.

Declerck, P.J., De Mol, M., Vaughan, D.E., Collen, D., 1992. Identification of a conformationally distinct form of plasminogen activator inhibitor-1, acting as a noninhibitory substrate for tissue-type plasminogen activator. J. Biol. Chem. 267, 11693-11696.

Dementiev, A., Dobo, J., Gettins, P.G., 2006. Active site distortion is sufficient for proteinase inhibition by serpins: structure of the covalent complex of alpha1proteinase inhibitor with porcine pancreatic elastase. J. Biol. Chem. 281, 34523457.
Dewilde, M., Strelkov, S.V., Rabijns, A., Declerck, P.J., 2009. High quality structure of cleaved PAI-1-stab. J. Struct. Biol. 165, 126-132.

Durand, M.K., Bodker, J.S., Christensen, A., Dupont, D.M., Hansen, M., Jensen, J.K., Kjelgaard, S., Mathiasen, L., Pedersen, K.E., Skeldal, S., Wind, T., Andreasen, P.A., 2004. Plasminogen activator inhibitor-I and tumour growth, invasion, and metastasis. Thromb. Haemost. 91, 438-449.

Egelund, R., Rodenburg, K.W., Andreasen, P.A., Rasmussen, M.S., Guldberg, R.E. Petersen, T.E., 1998. An ester bond linking a fragment of a serine proteinase to its serpin inhibitor. Biochemistry 37, 6375-6379.

Emsley, P., Cowtan, K., 2004. Coot: model-building tools for molecular graphics. Acta Crystallogr. D. Biol. Crystallogr. 60, 2126-2132.

Engh, R.A., Schulze, A.J., Huber, R., Bode, W., 1993. Serpin structures. Behring Inst. Mitt. 93, 41-62.

Erickson, J., Neidhart, D.J., VanDrie, J., Kempf, D.J., Wang, X.C., Norbeck, D.W., Plattner, J.J., Rittenhouse, J.W., Turon, M., Wideburg, N., 1990. Design, activity, and 2.8 A crystal structure of a C2 symmetric inhibitor complexed to HIV-1 protease. Science 249, 527-533.

Gils, A., Declerck, P.J., 1997. Proteinase specificity and functional diversity in point mutants of plasminogen activator inhibitor 1. J. Biol. Chem. 272, $12662-12666$

Gils, A., Knockaert, I., Declerck, P.J., 1996. Substrate behavior of plasminogen activator inhibitor-1 is not associated with a lack of insertion of the reactive site loop. Biochemistry 35, 7474-7481.

Gils, A., Meissenheimer, L.M., Compernolle, G., Declerck, P.J., 2009. Speciesdependent molecular drug targets in plasminogen activator inhibitor-1 (PAI1). Thromb. Haemost. 102, 609-610.

Hekman, C.M., Loskutoff, D.J., 1985. Endothelial cells produce a latent inhibitor of plasminogen activators that can be activated by denaturants. J. Biol. Chem. 260 11581-11587.

Huntington, J.A., Read, R.J., Carrell, R.W., 2000. Structure of a serpin-protease complex shows inhibition by deformation. Nature 407, 923-926.

Jensen, J.K., Gettins, P.G., 2008. High-resolution structure of the stable plasminogen activator inhibitor type-1 variant 14-1B in its proteinase-cleaved form: a new tool for detailed interaction studies and modeling. Protein Sci. 17, 1844-1849.

Juhan-Vague, I., Alessi, M.C., Declerck, P.J., 1995. Pathophysiology of fibrinolysis. Baillieres Clin. Haematol. 8, 329-343.

Kabsch, W., 1993. Automatic processing of rotation diffraction data from crystals of initially unknown symmetry and cell constants. J. Appl. Crystallogr. 26, 795800.

Kruithof, E.K., Tran Thang, C., Ransijn, A., Bachmann, F., 1984. Demonstration of a fast-acting inhibitor of plasminogen activators in human plasma. Blood 64 , 907-913.

Lijnen, H.R., 2005. Pleiotropic functions of plasminogen activator inhibitor-1. J. Thromb. Haemost. 3, 35-45.

McCoy, A.J., Grosse-Kunstleve, R.W., Adams, P.D., Winn, M.D., Storoni, L.C., Read, R.J. 2007. Phaser crystallographic software. J. Appl. Crystallogr. 40, 658-674.

Mestas, J., Hughes, C.C., 2004. Of mice and not men: differences between mouse and human immunology. J. Immunol. 172, 2731-2738.

Mottonen, J., Strand, A., Symersky, J., Sweet, R.M., Danley, D.E., Geoghegan, K.F. Gerard, R.D., Goldsmith, E.J., 1992. Structural basis of latency in plasminogen activator inhibitor-1. Nature 355, 270-273.

Munch, M., Heegaard, C.W., Andreasen, P.A., 1993. Interconversions between active, inert and substrate forms of denatured/refolded type-1 plasminogen activator inhibitor. Biochim. Biophys. Acta 1202, 29-37.

Murshudov, G.N., Vagin, A.A., Dodson, E.J., 1997. Refinement of macromolecular structures by the maximum-likelihood method. Acta Crystallogr. D. Biol. Crystallogr. 53, 240-255

Nar, H., Bauer, M., Stassen, J.M., Lang, D., Gils, A., Declerck, P.J., 2000. Plasminogen activator inhibitor 1 . Structure of the native serpin, comparison to its other conformers and implications for serpin inactivation. J. Mol. Biol. 297, 683-695.

Narayanaswamy, M., Wright, K.C., Kandarpa, K., 2000. Animal models for atherosclerosis, restenosis, and endovascular graft research. J. Vasc. Interv. Radiol. 11, 5-17.

Ngo, T.H., Zhou, Y., Stassen, J.M., Declerck, P.J., 2002. Importance of N-terminal residues in plasminogen activator inhibitor 1 on its antibody induced latency transition. Thromb. Haemost. 88, 288-293.

Prins, M.H., Hirsh, J., 1991. A critical review of the evidence supporting relationship between impaired fibrinolytic activity and venous thromboembolism. Arch. Intern. Med. 151, 1721-1731.

Sharp, A.M., Stein, P.E., Pannu, N.S., Carrell, R.W., Berkenpas, M.B., Ginsburg, D. Lawrence, D.A., Read, R.J., 1999. The active conformation of plasminogen activator inhibitor 1, a target for drugs to control fibrinolysis and cell adhesion. Struct. Fold. Des. 7, 111-118.

Shore, J.D., Day, D.E., Francis Chmura, A.M., Verhamme, I., Kvassman, J., Lawrence, D.A., Ginsburg, D., 1995. A fluorescent probe study of plasminogen activator inhibitor-1. Evidence for reactive center loop insertion and its role in the inhibitory mechanism. J. Biol. Chem. 270, 5395-5398.

Stein, P.E., Baek, K., 2002. 1.8 Å resolution structure of latent plasminogen activator inhibitor-1. Protein data bank code 11j5. Ref Type: Unpublished Work.

Stout, T.J., Graham, H., Buckley, D.I., Matthews, D.J., 2000. Structures of active and latent PAI-1: a possible stabilizing role for chloride ions. Biochemistry 39 , 8460-8469.

van Meijer, M., Pannekoek, H., 1995. Structure of plasminogen activator inhibitor 1 (PAI-1) and its function in fibrinolysis: an update. Fibrinolysis 9, 261-276.

Wiman, B., 1999. Predictive value of fibrinolytic factors in coronary heart disease. Scand. J. Clin. Lab. Invest. 59, 23-31. 
Xu, Z., Balsara, R.D., Gorlatova, N.V., Lawrence, D.A., Castellino, F.J., Ploplis, V.A., 2004. Conservation of critical functional domains in murine plasminogen activator inhibitor-1. J. Biol. Chem. 279, 17914-17920.

Xue, Y., Björquist, P., Inghardt, T., Linschoten, M., Musil, D., Sjölin, L., Deinum, J., 1998. Interfering with the inhibitory mechanism of serpins: crystal structure of a complex formed between cleaved plasminogen activator inhibitor type 1 and a reactive-centre loop peptide. Structure 6, 627-636.

Zhou, A., Huntington, J.A., Pannu, N.S., Carrell, R.W., Read, R.J., 2003. How vitronectin binds PAI-1 to modulate fibrinolysis and cell migration. Nat. Struct. Biol. 10, 541-544. 